\title{
Donation in 1866. Names of Members
}

\section{Lieut. H. L. Powys-Keck}

To cite this article: Lieut. H. L. Powys-Keck (1867) Donation in 1866. Names of Members, Royal United Services Institution. Journal, 10:S1, XV-XV, DOI: 10.1080/03071846709417984

To link to this article: http://dx.doi.org/10.1080/03071846709417984

\section{曲 Published online: 25 Sep 2009.}

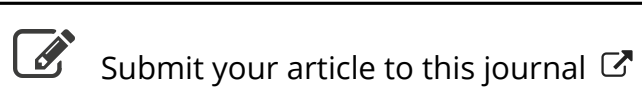

\footnotetext{
山 Article views: 2
}

Q View related articles $\longleftarrow$ 


\section{DONATION IN 1866.}

Porrys-Keck, H. I., Iient., Leicester Yeomanry Caralry, $5 l$.

\section{NANES OF MEMBERS}

WHO JOINED TIIE INSTITUTION BETWEEN THE 16TH JULY AND

31ST DECEMEBR, 1866.

IIIFE.

Holdich, F. A, C.B., Col. 1st Bat. 20th Regt., $0 l$.

Farquhar, Harry, R., Lieut. 1st Battalion 21th Foot, $9 l$.

Harrey, J. W. F., Lieut., R.N., $9 l$.

Gleichen, The Count, Capt. R...., $9 l$.

Arontgomerie, O. T. MI, Lieut. Gren. Guards, 0l.
Hubbard, C. G., Iieut. Gren. Gds $0 l$.

Mrainwaring, $\mathrm{K}$ H., Licut. R.N., 9 l.

Edinburgh, $\cdot$ H. R. H. Duke of, K.G. Capt. R.N., $9 l$.

Tieal, Wm.; Capt. 52nd Regt., $9 l$.

Saltmarshe, A., Major 70th Regt., 97 .

Elgec, Charles, Lieut.-Coì. 2urd Royal Welch Fusiliers, $0 l$.

\section{ANXUAI.}

Macneil, Hector A., Capt. Edinburgh 2nd Regt. Light Inf. Mil., Il.

Willis, Clarles W., Capt. 33rd Regt., $1 l$.

IIccGwire, E.T. St. L., Capt. 1st Royals, 12.

Buller, E. W., Lieut. R.A., $1 l$.

Jocelyn, Hon. John, Capt. late 7th Hus.

Gregrory, G. B., Capt. lato 37th Mid. Rifle Vols., 1 .

Berthon, Alderson, Licut. Isle of Wight Art. Mil. $1 l$.

Garlick, Jolın M., Licut. 106th Regt.

Pye, Kellow, Charles, Lieut. R.E., 11 .

Simpson, Cortland H., Capt. R.N., 1 .

Roe, E. M., Tieut. 23rd R. W. Fus., $1 l$.

Jones, Douglas F., Lient. R.A., $1 l$.

Squirl, Wrm., Capt. 91st Highlrs., 11.

Ilome, R. II., Lieut. 13th Light Inf., $1 l$.

Cautler, Henry, Lieut. R.E., $1 l$.

Grant, W. J. E., Licut.-Col. R.A, 1 l.

Russcll, Godfrey, Capt. Shropshire Jil.,1l.

Evans, F. T., Ensign Ceslon Rilles, 1 .

IIethuen, Hon. Paul S., Lieut. Scots Fus. Gids.

Riviere, Frederick, Capt. IIon. A. Co.

Iegge, Compton, Lieut. Oxford Mil., 11.

Brome, G. A., Capt. 1st Bengal Natire Inf., 1 .

Custance, F. II., Licut. Gren. Gds., 1 l.

Tew, Cyril B., Licut. 6Sth J.t. Inf., $1 l$.

Allenson, $A$. C., Lieut. 1st W. I. Regt., $1 l$.

Williams, J. Aubrey, Ensign Goth Rogal Riffes, 11 .
Caulfield, Alexander, Capt. Greu. Gds. Carington, Hon. W. II. P., Licut. Gren. Gds.

Lascelles, II. UlickTiscount, Licut. Gren. Gds.

Van do Werer, A. S. B., Lt. Gren. Gds.

Thornton, E. Z., Lievt. 10 th Regt., 1 .

Ricketts, Geo. Thorne, Lieut. late 5th Dr. Gds., $1 l$.

Wilson, C. P., Licut. II. MI. Indian Nary, 12.

Follett, R. W. Webb, Lieut. Colds. Gds., 12.

Mracdonald, N., Capt. 5th Fus., 1 l.

Hotlgson, Geo. E., Capt. 41th Regt., 11.

Cochrane, W. M., Lt.-Col. 2nd Adm. Bat. Surrey Rifle Yols., Capt.1stLanark Aril., 11.

Allix, Foel C., Liout. Gren. Gds., 1 .

Corkran, Charles S., Lt. Gren. Gds., 1 l.

Resaardson, O. B., Lieut. Gren. Gds., $1 l$.

Fleteher, T. C., Capt. R.A., 1 l.

Loctner, Chris. P., Capt. 36th Middlescx Riflo Tols., il.

Todd, T. Fentham, Ens. 62nd Regt., 1 .

Hildyard, R. C. T., Lieut. R.E., 1 .

Tounochy, Talens, Capt. 81st Regt., $1 l$.

Bulger, O. O., Lieut. 4th W. I. Regt., 1 l.

Paget, II. E. Lisut. 87th Roy. Irish Fus. il.

Greares, G. R., It.-Col. 70th Regt. 1 .

Whitmore, I. A., Col. Assist. Adj.-Gen. Horso Gds., $1 l$. 